\title{
GEOGRAPHICALLY DiSTRIBUTED "MULTI-OPERATOR" CONTROL FOR MECHATRONIC DEVICES VIA THE INTERNET
}

\author{
Victor Andreev ${ }^{\mathrm{a}, \mathrm{b}, \mathrm{d}}$, Kirill Kirsanov ${ }^{\mathrm{b}, \mathrm{c}, \mathrm{d}}$, Valentin Pryanichnikov ${ }^{\mathrm{a}-\mathrm{e}}$ \\ "MSTU “STANKIN", Vadkovsky lane 1, Moscow, 127994, Russia \\ ${ }^{b}$ International Institute of New Educational Technologies, RSUH, Miusskaya sq. 6, Moscow, 125993, Russia \\ ${ }^{c}$ Keldysh Institute of Applied Mathematics, RAS, Miusskaya sq. 4, Moscow, 125047, Russia \\ 'International Laboratory "Sensorika”, Miusskaya sq. 4, Moscow, 125047, Russia \\ ${ }^{e}$ University of Zadar, Mihovila Pavlinovica bb, Zadar, 23000 Croatia
}

\begin{abstract}
A technology of creating of geographically distributed multi-operator control of mechatronic devices via the Internet is described. We propose a hierarchical structure for the management of a group of robotized systems by combining their information-measuring and control systems into a local area network with mobile nodes via the Internet. A hardware and software architecture is proposed for interaction between mechatronic devices of various models and manufacturers. This mechanism is based on the concept of "drivers". Requirements to the hardware and software platform have been developed for the combination into a group of robotized systems. Reference realizations of protocols for message exchange and drivers for x86 and Arduino platforms have been created. We have developed graphical user interfaces for all components of the system. This software enables automatic configuration of the geographically distributed network and a full-featured access via the Internet to specific robotized devices (including to individual components of a mobile robot). The hardware and software system created on the basis of the proposed technology provides real-time feedback (including visual feedback) between a control object and an operator and makes it possible to remotely monitor the program code execution on the onboard computer (including microcontrollers) and dynamically correct it without rebooting the system. We describe the technical implementation of a pilot project for the hardware and software system by the example of supervisory (via the Internet) control of AMUR (IL "Sensorika") and Robotino (Festo) educational robots with a video surveillance of command execution.
\end{abstract}

Keywords: robotic system; mobile robot; mechatronic device; multi-operator control; geographically distributed control; local area network
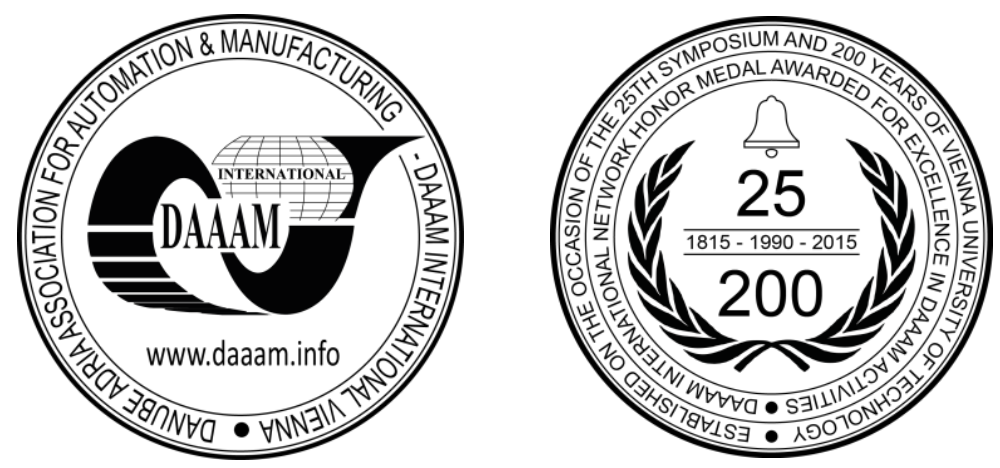

This Publication has to be referred as: Andreev, V[ictor]; Kirsanov, K[irill] \& Pryanichnikov, V[alentin] (2016). Geographically Distributed "Multi-Operator" Control for Mechatronic Devices via the Internet, Proceedings of the 26th DAAAM International Symposium, pp.0004-0011, B. Katalinic (Ed.), Published by DAAAM International, ISBN 9783-902734-07-5, ISSN 1726-9679, Vienna, Austria DOI:10.2507/26th.daaam.proceedings.001 


\section{Introduction}

The software designed for the interaction of application software with the network and unifying the interaction between programs in heterogeneous computing platforms is termed as middleware [1]. This kind of software serves as a subsystem of larger software. Middleware has been intensively studied in modern database and Internet technologies; however, these studies for robotics have not reached the same level. Most likely, this is associated with the fact that until recently the software for robotic systems has been rather simple (in terms of software engineering) and has not gone beyond simple interactions between control objects.

Spatially distributed control of a group of mobile robotic devices can be implemented on the basis of wellknown frameworks such as the Robotics Operating System (ROS) [2] and Microsoft Robotics Developer Studio (MRDS) [3], and their precursors such as Player Project (software for research into robotics and sensor systems), LAAS GenoM (a tool for designing real-time software architectures), and URBI (an open-source operating system for robots). These systems can be roughly classified as "large", i.e., comprising many components to solve various problems: SLAM (Simultaneous Localization and Mapping), calibration of sensors, image processing, etc. However, there also exist "small" robotic frameworks, such as Concurrency and Coordination Runtime (included in MRDS) providing merely message passing between components.

These software systems can efficiently solve the task but merely within a single territory (laboratory, production unit, plant); i.e., they do not solve the problem of geographically distributed control. Consequently, these software systems have no mechanisms of control (via the Internet) of mechatronic devices with a visual feedback to monitor the execution of commands and control programs, which is particularly critical in supervisory control systems. The peculiarity of this method of control is to organize the transfer via Internet multistream video and command/programs of control taking into account network latency and non-guaranteed delivery times of packets.

These systems are designed for operating in a local area network (LAN) with a stable communication channel and have almost no tools designed for correct failure recovery in the data transmission channel. But this problem arises whenever mobile robotic systems (MRSs) are to be operated when the communication channel includes a radio channel; this raises the problem of ensuring the system stability to disruption of communication. It is also necessary to ensure a high performance in the transmission of both long (for example, multistream video) and short messages.

In a geographically distributed system, when control of "foreign" mechatronic devices is permitted, it becomes important to have a mechanism of the distribution of access rights; this mechanism is missing in ROS and MRDS.

It is known that distant control of mechatronic devices can be implemented in practice via the Internet and radio channel with a feedback [4, 5]. The project was accomplished in 2009-2011 in the Russian State Scientific Center for Robotics and Technical Cybernetics (RTC) and the Rocket and Space Corporation Energia (Russia), jointly with the Institute of Robotics and Mechatronics of the German Aerospace Center (DRL-RMC). The project investigated the possibility of using the Internet for controlling from the Earth a ROBOTIC two-link robot mounted on the outer surface of the Russian segment of the International Space Station (ISS). The communication sessions with the ISS were provided from both DRL-RMC (Germany) and RTC (St. Petersburg, Russia) through DRL-RMC using the Internet for the communication between RTC and DRL RMC. The project had the specific feature that a human-machine interface with torque/force feedback is used. This project considered only the influence of delays in communication channels on the process of control.

Thus, the technology of geographically distributed control of a group of MRSs requires the development of a qualitatively new software architecture. Below, we consider the problem of geographically distributed control (via the Internet) of a unified group of robotic systems consisting of mobile robots (MRs) of different models and manufacturers. In this case, MRs operators can be located on geographically remote territories.

\section{Geographically distributed control of a group of mobile robots}

Digital representation of data in the information-measuring and control system (IMCS) of a mobile robotic system makes it possible to combine its electronic and mechatronic units into a local area network with mobile nodes [6]. This method of combination allows the network features to be extended to the structure of the IMCS of both an individual robotic system and several robotic systems on the basis of network technologies. In our case, the most important features are reconfigurability and scalability. These features imply that one can easily extend or modify any resources of the robotic system (sensors, calculators, microprocessors, radio channel generators, and other IP-systems) and specifically combine the IMCSs of multiple mobile robotic systems into an interconnected geographically distributed system [7].

The use of network technologies makes it possible to arrange a geographically distributed "multi-operator" control of a group of MRSs. The term "multi-operator" means that individual robotic devices involved in the group can be controlled by operators located in different territories. Under certain conditions, this control allows for the one-tomany mode, i.e., if granted with the appropriate right, one operator can interfere into the operation of electronic and mechatronic devices in any robotic systems involved in the group (including dynamic reprogramming of computing devices). An example of this structure for controlling a group of MRs is shown in Fig. 1. 




Fig. 1. Geographically distributed "multi-operator" control of a group of mobile robots

The communication system designed for control (via the Internet and radio channels) of a group of mobile mechatronic devices must have the following properties:

- provide both "direct" (from onboard TV-cameras) and "alien" (external view from the MR operational range) visual feedback for remote monitoring over the execution of commands and control programs;

- be stable to disruption of communication;

- provide high performance in the transmission of both long and short message.

- We consider hardware and software solutions ensuring these properties. The main requirements for a multicomponent robotic system are as follows [8]:

- the electronic and mechatronic components of the IMCS of the robotic system must be elements of a local area network with mobile nodes;

- the communication between mobile and stationary units of the robotic system must be arranged through digital radio channels (Wi-Fi, Wi-Max, etc.);

- the radio channel range should be increased and its quality should be improved through the construction of an optimal operational remotely controlled architecture of the distributed IMCS;

- it is necessary to use a multi-camera system of technical vision: multiple TV-cameras (including those with the PTZ (pan-tilt-zoom) functionality) onboard the mobile unit of the robotic system and cameras on escort Satellitedevices (stationary or mounted on mobile platforms);

- video signals and signals from other sensors must be preprocessed in a stand-alone mode onboard the mobile unit of the robotic system (digitization, noise filtering, error correction, image compression, etc.);

- operational remote monitoring (with a control desk) of the power system and the radio channel quality is mandatory;

- the remote control desk of the operator must provide:

- continuous (with a standard frame rate) display of video streams from all TV-cameras as raster images;

- mnemonic display (on the monitors) of indications of other sensors and the state of controlled parameters (including audio alarm at critical values);

- possibility of remote control of TV-camera functions (such as PTZ) and LAN settings (for example, the choice of an algorithm for the compression of images or its parameters);

- possibility of remote monitoring of the program code execution on the onboard computer and its dynamical correction without rebooting the system;

- the communication channels (both the Internet and radio channels) must be protected from unauthorized access (for example, by using VPN-tunnel).

A structure that satisfies the above-mentioned requirements was implemented as a specialized software and hardware system designed for supervisory control of a group of educational mobile robots. The networking mechanism developed by us was used to organize full-featured access (via the Internet) to mechatronic devices of different types. This mechanism is based on the concept of "drivers". 


\section{The concept of "drivers"}

Minimal control object in the proposed structure is any electronic or mechatronic device, such as an onboard computer, a microcontroller, a sensor, a manipulator, or another actuator. Therefore, a key problem in this case was to develop a unified system of software construction rules for specific models of robotic devices, allowing mechatronic components to be included into the LAN without remodeling their low-level code. In other words, it was required to develop a finite set of program instructions of control and network protocols for building an application programming interface (API). This interface must provide a transparent integration of the elements of the mechatronic device and its software into the information space of the global IMCS.

We implement this API on the basis of the concept of "drivers": for each mechatronic device, a unified network control protocol must be built on the base of existing low-level APIs. Then, the manufacturer of mechatronic devices can relatively easily integrate its own device into the network by constructing only an additional driver that implements the protocol-induced program instructions of control and the networking protocols.

When a new mechatronic device appears in the network, the software of the control subsystem automatically adds this device into the shared network during the interaction with the driver. Simultaneously, the available mechatronic devices and their descriptions are written into the general catalog of technical specifications.

The driver of each mechatronic device on the onboard computer of the MR is a separate computing process, and the joint operation of these processes should be arranged and synchronized. To solve these problems, we use the following approaches:

- $\quad$ Each command or data from sensors are supplied with a time marker when passing each node of the LAN.

- Each driver contains its own copy of the "name server", which stores information about neighboring (in terms of the network topology) drivers.

- Each driver involves a dynamically generated interaction graph, which stores information about neighbors.

- A textual (ASCII) format is used for data exchange.

- Data exchange makes it possible to arrange the transmission of both individual commands and entire programs.

Supplying messages with a time marker allows one to avoid violation of the sequence of events in case of failures, correctly synchronize the operation of drivers, and take into account the delays and the time difference between several geographically distributed network nodes. The vector clock algorithm allows for synchronization independent of the real-time clock on onboard computers.

The "name server" is a specialized software that scans the network every 20 seconds and looks for similar services. When such services have been detected, the "name server" exchanges all available data with them using an algorithm close to the distributed hash tables (DHT). This gives rise to the quality of decentralization. For example, a robotic system configured within a specific LAN can be disconnected from it without changes and without affecting the normal operation of the robotic system.

The interaction graph is a data structure that stores information about neighboring (in terms of the network topology) drivers and their interactions. The interaction graph is automatically recovered after communication breaks even if the network topology is slightly changed. This ensures that the network data topology can be recovered after its destruction caused by failures in communication channels. This gives rise to the quality autoconfiguration, which makes it possible to dynamically modify the topology of the geographically distributed IMCS of the group of mobile robots but within the same network.

The textual format of data exchange makes it possible to simplify the debugging of the software and arrange an interaction with the system without additional libraries, which is extremely important in problems of integration.

To integrate the robotic system into a geographically distributed group, it was necessary not only to arrange the interaction between electro-mechanical components of the robot and the onboard computer, but also to integrate its IMCS directly into the general control hierarchy.

\section{The hardware and software architecture for interaction mechatronic devices}

The hardware and software system was implemented with the following properties:

- universality: integration of robotic and other mechatronic systems of different models and manufacturers into the system;

- network reliability: guaranteed delivery of packets between control interfaces of the system at all networking levels;

- integrity: a mechanism of control for access rights and delineation of privileges between users;

- flexibility and scalability: decentralized control of the network structure, its on-line configurability;

- $\quad$ security: protection from unauthorized access to control interfaces.

The data exchange between the distributed IMCS elements was arranged on the basis of well-known network technologies and solutions with synchronous and asynchronous interaction based on the TCP\IP protocol stack. It contains the following components: 
- $\quad$ protocols of synchronous and asynchronous messaging;

- $\quad$ unified directory of services;

- $\quad$ program unit of operator.

According to the classification of the well-known OSI model, the interaction in our case occurs at the application level, which can be conditionally divided into two sublevels: transport and logical.

The transport sublevel should meet the following requirements:

- high performance in the transmission of both long (for example, multi-stream video; megabytes) and short (for example, control commands; bytes) messages;

- $\quad$ stability to connection breaks (for example, because of the unstable operation of the radio channel);

- possibility of arranging a one-to-many interaction, which is necessary for group control.

The logical sublevel should meet the following requirements:

- be cross-platform and multilingual: the possibility of working on different operating systems (Windows, Linux x86164larm, Android, MCST Elbrus) and in different languages (C, Java, Python, Fortran);

- human-readability: capability to perceive the information received without using special-purpose program tools (this is achieved by data transmission in a textual format, except for cases when this is critical to the performance; for example, in the case of video stream transmission);

- the possibility of isolated operation: the performance of interacting mechatronic devices involved in a LAN of a given hierarchy level should not be violated when these devices are disconnected from a higher level network that is external to them;

- decentralization and capacity for self-organization: there is no need for the existence of a "central" server regulating the operation of all components of the system, except in cases when the access rights are restricted and users are registered.

To build a formal description of interaction in multi-agent network, we used the actor model [9]. The mechanism for message transmission is based on the ZeroMQ library [10], which serves as a high-level "wrapper" over standard network sockets of the operating system. This mechanism includes a number of features such as the automatic recovery of communication after failure without loss of the context, the possibility of tunneling through the low-layer protocols of UDP, TCP, and IPC, or PGM (Pragmatic General Multicast). In addition, unlike more complex protocols (such as Concurrency and Coordination Runtime), ZeroMQ is sufficiently simple, which allowed it to be implemented directly on the Arduino microcontroller with an Ethernet Shield module, but with some limitations due to its low performance. For example, the maximum frequency of command processing in a test run with this microcontroller was $20 \mathrm{~Hz}$.

Figure 2 shows the general schematic of the interaction: two mechatronic devices RC1 and RC2 (RobotComponent) pass the registration procedure asynchronously in the name server NS (NameServer). Then, the control system CS (ControlSystem) requests the list of registered devices to perform synchronous and asynchronous interaction with mechatronic devices. In this case, the name can be an IP-address and its port, an arbitrary text (for example, "engine") or a full path in the hierarchy (for example, "University $\rightarrow$ Laboratory $1 \rightarrow$ Robotino $\rightarrow$ Engines $\rightarrow 3 \rightarrow$ PWM".

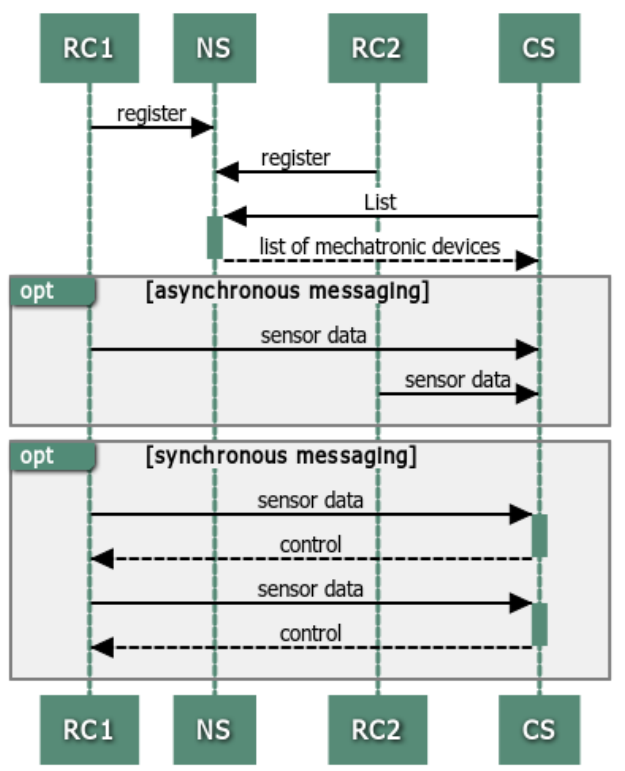


Fig. 2. Mechanism of network interaction in the proposed architecture

The driver of each device provides:

- $\quad$ a naming and identification mechanism (way of accessing the device by its name);

- a command sending and receiving, including by another driver.

The messaging scheme is shown in Fig. 3. The control commands and data are transmitted in the JSON format, which is sufficiently simple and can be implemented on low-power processors including AVR (Arduino).

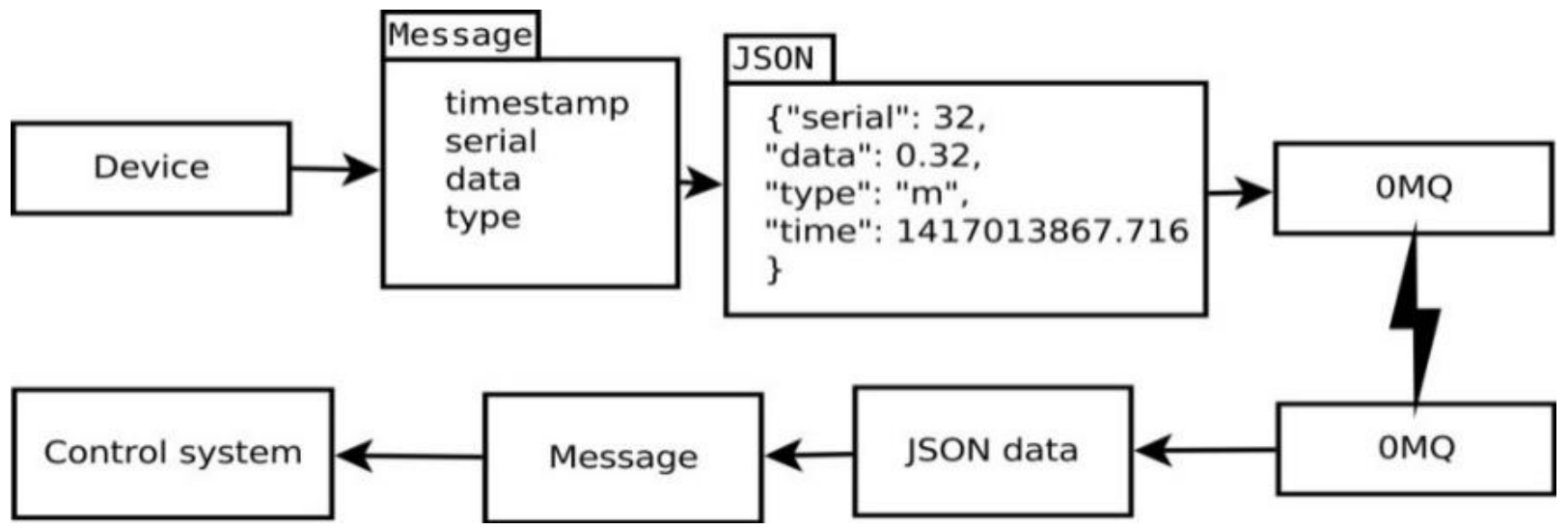

Fig. 3. Schematic of message transmission: Device generates Message, which is encoded into the JSON format and transmitted to OMQ (ZeroMQ)

The JSON is a textual (ASCII) format for structured data, capable of serializing (i.e., representing as a sequence of bytes) lists (vector, array) and associative arrays (i.e., sets of "key-value" pairs), which makes it possible to manually generate control commands without specialized libraries, as required in other similar systems. In addition, this solution considerably simplifies the use of multiple programming languages or software and hardware platforms. This is achieved by the fact that JSON and ZeroMQ parsers have already been implemented for the vast majority of platforms and languages.

Also, it becomes possible to supply the transmitted data with additional information without modifying their host software or arbitrarily change the order of the fields in a single message. This makes it possible to arrange the transmission of both individual commands and entire programs, which are executed (immediately after being received) by an interpreter available in onboard computers [11]. In this way, one can arrange dynamic reprogramming of a mechatronic device; i.e., without stopping or "rebooting" onboard computing devices of a MR, one can update its software or adjust its operation. This considerably simplifies the concurrent support for different versions of a driver in a single network of mechatronic devices in problems of geographically distributed control.

The JSON parser is a standard library in many programming languages. However, for AVR, we had to develop its own parser that implements merely a minimum required subset of JSON without support of arbitrary nested structures but with a satisfactory performance $(40 \mathrm{~Hz})$.

The information redundancy arising from its textual representation has no significant effect on the data throughput because the commands are usually compact even in a textual representation and can be compared in size with the IP packet header ( 20 bytes).

However, this protocol is inefficient for transmitting multichannel video; therefore, well-proven protocols such as RTSP (Real Time Streaming Protocol) and M-JPEG over HTTP are used in this case.

To integrate a mechatronic device into a geographically distributed system, one should ensure merely that the following two basic requirements to the hardware and software platform of the device are met:

- the device must have a LAN interface;

- the device must have a microcontroller and the ability to install the software implementing the minimum necessary subset used in the protocol, i.e. ZMQ.

In line with the above-mentioned requirements, the authors have developed a driver for the Arduino microcontroller. Additionally, the iPython Notebook software was used [12]. This made it possible to interactively modify the software running on the robot. proposed.

A more detailed description of this approach can be found in [13]. Also, the direction for creation the driver is

To visualize the components detected in the network, we developed control software for the operator screen. Interface was created by the control software, which represents all detected components as a tree (see Fig. 4). 


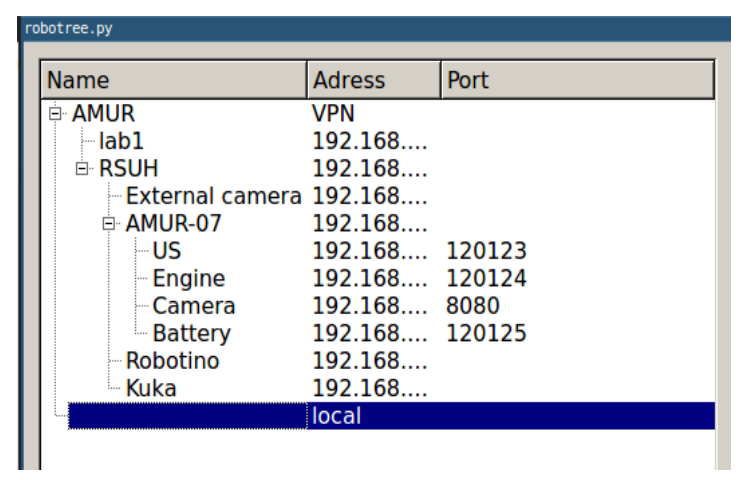

Fig. 4. One of the windows of the screen interface. Tree representation of AMUR components: ultrasonic sensors (US), engines (Engine), TV-camera (Camera), and rechargeable battery (Battery)

\section{Conclusion}

The proposed structure of geographically distributed control (via the Internet) of a group of mobile robotized devices was implemented as a hardware and software system and proved its efficiency for creating operational channels of multi-operator control of AMUR (Autonomous Mobile Educational Robot, IL "Sensorika", Russia) and Robotino (Festo concern, Germany) type educational robots. These VPN-tunnels are currently operating between the MSTU "STANKIN" (Moscow) and the Center for Technological Support of Education of the International Institute of New Educational Technologies (CTSE IINET) of Russian State University for the Humanities (RSUH, Moscow). A similar communication channel also exists between the Keldysh Institute of Applied Mathematics, Russian Academy of Sciences (Moscow), CTSE IINET RSUH, and the Far East Federal University (Vladivostok).

This geographically distributed network structure of the information-measuring and control system ensures that the multidisciplinary experts (including representatives of manufacturers) that are far from the place of activities can be simply engaged in the mobile robot control.

This information and communication infrastructure can be used in the system of distance education [8] for arranging student access to real mechatronic systems located at remote areas (specifically, for connecting branches to laboratory facilities of the main educational institution). This makes it possible to concentrate the technical resources in one place and organize the education of student groups remotely (from branches) without reducing the education quality. In addition, this structure allows geographically dispersed research teams to be combined around an entity that has appropriate facilities and resources, or mechatronic devices of different groups to be combined into a single system with any-to-any access.

Also, Bionic Assembly System is a very good example of the possible use of the proposed network solutions [16, 17].

\section{Acknowledgements}

This study work was supported by the Russian Academy of Engineering, project "Intelligent Robotronics" and partially by the Russian Foundation for Basic Research, project nos. 13-07-01032 and 13-07-00988.

\section{References}

[1] Wolfgang Emmerich, Mikio Aoyama, Joe Sventek. The impact of research on the development of middleware technology. ACM Transactions on Software Engineering and Methodology. - N. Y.: ACM, 2008. - V.17. - No 4. - P.19- 48 .

[2] ROS. - URL: http://www.ros.org. Accessed: 17.09.2015.

[3] Microsoft Robotics Developer Studio 4. - URL: http://www.microsoft.com/enus/download/details.aspx?id=29081. Accessed: 17.09.2015.

[4] Günter Niemeyer and Jean-Jacques E. Slotine. Telemanipulation with time delays. Int. Journal of Robotics Research, 23(9):873-890, September 2004.

[5] Jordi Artigas, Ryu Jee-Hwan, C.Preusche and G.Hirzinger. Network representation and passivity of delayed teleoperation systems. Intelligent Robots and Systems (IROS), 2011 IEEE/RSJ International Conference on 25-30 Sept. 2011, pp.177-183.

[6] Pryanichnikov V., Andreev V. The Application of Network Technologies to Constructing Group Controlled Systems with Machine Vision for Mobile Robots. Annals of DAAAM for 2012\& Proceedings of the 23th international DAAAM Symposium "Intelligent Manufacturing \& Automation" 24-27th October 2012 Zadar, Croatia, ISSN 2304-1382, 2012. - V.23, No.1, - pp.1167-1174.

[7] V.P.Andreev, K.B.Kirsanov, P.F.Pletenev, Yu.V.Poduraev, V.E.Pryanichnikov, E.A.Prysev. Technology Supervisory Control for Mechatronic Devices via the Internet. 25th DAAAM International Symposium on Intelligent Manufacturing and Automation, DAAM 2014. Procedia Engineering, 2015. - V.100, - pp.33 - 40.

[8] Andreev V., Pryanichnikov V., Poduraev Y., Kuvshinov S. Education on the basis of virtual learning robotics laboratory and group-controlled robots. 24th DAAAM International Symposium on Intelligent Manufacturing and Automation, DAAM 2013. Procedia Engineering, 2014. - V.69. - P.35 - 40. 
[9] Hewitt Carl, Peter Bishop, and Richard Steiger. A universal modular actor formalism for artificial intelligence. In Proceedings of the 3rd international joint conference on Artificial intelligence. Morgan Kaufmann Publishers Inc., 1973. - P. 235-245.

[10] Hintjens Pieter, ZeroMQ: Messaging for Many Applications, O'Reilly Media, Inc., 2013.

[11] Kirsanov Kirill. The Architecture of Robotics Control Software for Heterogeneous Mobile Robots Network. 24th DAAAM International Symposium on Intelligent Manufacturing and Automation, DAAM 2013. Procedia Engineering, 2014. - V.69. - P.216 - 221.

[12] Python. - URL: https://www.python.org. Accessed: 17.09.2015.

[13] Andreev V., Pletenev P. The method of network control of robotic systems of different models and manufaturers. 26th DAAAM International Symposium on Intelligent Manufacturing and Automation, 21-25th October 2015 Zadar, Croatia.

[14] ØMQ Licensing. - URL: http://zeromq.org/area:licensing. Accessed: 17.09.2015.

[15] GNU lesser general public license. Version 3, 29 June 2007. - URL: http://www.gnu.org/licenses/lgpl.html. Accessed: 17.09.2015.

[16] Katalinic B., Kukushkin I., Pryanichnikov V., Haskovic D. Cloud Communication Concept for Bionic Assembly System. Procedia Engineering, 1877-7058, 69 (2014) (2014), pp. 1562-1568 doi:10.1016/j.proeng.2014.03.156.

[17] Katalinic B., Kukushkin I., \& Haskovic D. Bionic Assembly System Cloud: Functions, Information Flow and Behavior. In 9th International Conference of DAAAM Baltic, Industrial Engineering, ISBN 978-9949-23-620-6, ISSN 2346-6138. pp. 103-108, ed. T.Otto, Tallinn, Estonia, 2014. 